\title{
Características da Carcaça de Novilhos 5/8 Nelore-3/8 Charolês Abatidos em Diferentes Estádios de Desenvolvimento ${ }^{1}$
}

\author{
Miguelangelo Ziegler Arboitte ${ }^{2}$, João Restle ${ }^{3}$, Dari Celestino Alves Filho4, Leonir Luiz \\ Pascoal $^{5}$, Paulo Santana Pacheco ${ }^{6}$, Diogo Carvalho Soccal ${ }^{7}$
}

\begin{abstract}
RESUMO - Foram avaliadas as características quantitativas das carcaças de novilhos 5/8 Nelore-3/8 Charolês terminados em confinamento até estes atingirem o peso médio de abate (P) de 425, 467 e $510 \mathrm{~kg}$. Os novilhos apresentaram ao início do confinamento idade média de 660 dias, peso de 361 kg e estado corporal de 2,9 pontos. A dieta oferecida, com relação volumoso:concentrado de 60:40 na base matéria seca (MS), continha 10,25\% de proteína bruta e 72,18\% de nutrientes digestíveis totais. O volumoso foi silagem de milho contendo 46,5\% de grãos na MS. O rendimento de carcaça fria ( $\hat{Y}=37,618+0,011 \mathrm{Pi}+0,028 \mathrm{P}$ ) aumentou linearmente com o P. No entanto, quando o rendimento de carcaça foi expresso em relação ao peso de corpo vazio (PCV) este não foi influenciado pelo P. A espessura de gordura subcutânea na carcaça fria ( $\hat{\mathrm{Y}}=-15,499-0,001 \mathrm{Pi}+0,047 \mathrm{P})$, expressa por $100 \mathrm{~kg}$ de carcaça fria $(\hat{\mathrm{Y}}=-1,724-0,006 \mathrm{Pi}+0,013 \mathrm{P})$ e PCV ( $\hat{Y}=-1,124-0,003 P i+0,008 P)$ aumentou com o avanço do P. A área do músculo Longissimus dorsi aumentou linearmente com o P ( $\hat{\mathrm{Y}}=-33,471+0,120 \mathrm{Pi}+0,121 \mathrm{P})$, no entanto, quando expresso por $100 \mathrm{~kg}$ de carcaça fria decresceu ( $\hat{\mathrm{Y}}=45,173-0,023 \mathrm{Pi}-0,028 \mathrm{P})$ e quando expresso por $100 \mathrm{~kg}$ de corpo vazio não foi influenciada pelo peso de abate. A porcentagem do corte serrote decresceu ( $\hat{\mathrm{Y}}=63,007-0,001 \mathrm{Pi}-$ 0,026P), enquanto a do costilhar elevou-se $(\hat{Y}=-3,053+0,005 \mathrm{Pi}+0,030 \mathrm{P})$ com o aumento do P. A espessura de coxão ( $\hat{\mathrm{Y}}=6,223+0,014 \mathrm{Pi}+0,0310 \mathrm{P})$, comprimento de carcaça ( $\hat{\mathrm{Y}}=58,564+0,0916 \mathrm{Pi}+0,075 \mathrm{P})$, perna $(\mathrm{Y}=28,326+0,061 \mathrm{Pi}+0,050 \mathrm{P})$ e perímetro de braço ( $\hat{Y}=9,173+0,053 \mathrm{Pi}+0,017 \mathrm{P}$ ) foram influenciados positivamente pelo aumento do peso de abate dos animais. A conformação da carcaça não foi alterada pelo peso de abate.
\end{abstract}

Palavras-chave: cortes comerciais, gordura subcutânea, Longissimus dorsi, peso de corpo vazio

\section{Carcass Characteristics of 5/8 Nellore-3/8 Charolais Steers Slaughtered at Different Maturity Stages}

ABSTRACT - The carcass quantitative characteristics of 5/8 Nellore-3/8 Charolais steers feedlot finished to reach slaughter weight (SW) of 425, 467 and $510 \mathrm{~kg}$, were studied. At the beginning of the feedlot the average age, weight and body condition were, respectively, 660 days, $361 \mathrm{~kg}$ and 2.9 points. The diet offered, with 60:40 roughage:concentrate relation, dry matter basis (DM), contained 10.25\% crude protein and $72.18 \%$ of total digestible nutrients. The roughage was corn silage with $46.5 \%$ of grain in the DM. Cold carcass dressing percentage showed linear relation with SW $(\hat{\mathrm{Y}}=37.618+.011 \mathrm{Pi}($ initial weight $)+.028 \mathrm{SW})$. However, when dressing percentage was expressed in relation to empty body weight (EB), it was not influenced by SW. Subcutaneous fat thickness expressed per cold carcass $(\hat{\mathrm{Y}}=$ $-15.499-.001 \mathrm{Pi}+.047 \mathrm{SW})$, per $100 \mathrm{~kg}$ of cold carcass weight $(\hat{\mathrm{Y}}=-1.724-.006 \mathrm{Pi}+.013 \mathrm{SW})$ and per $100 \mathrm{~kg}$ of EB $(\hat{\mathrm{Y}}=-1.124-.003 \mathrm{Pi}+.008 \mathrm{SW})$ increased as SW increased. Longissimus dorsi area increased linearly with SW $(\hat{\mathrm{Y}}=-33.472+.120 \mathrm{Pi}+.120 \mathrm{SW})$, however, when expressed per $100 \mathrm{~kg}$ of cold carcass weight it declined ( $\hat{\mathrm{Y}}=45.173-.023 \mathrm{Pi}-.028 \mathrm{SW}$ ), and when expressed per $100 \mathrm{~kg}$ of EB, it did not show relationship with SW. The percentage of sawcut declined ( $\hat{Y}=63.007-.001 \mathrm{Pi}-.026 \mathrm{SW})$ while the side cut increased $(\hat{\mathrm{Y}}=-3.054+.005 \mathrm{Pi}+.030 \mathrm{SW})$, as SW increased. Slaughter weight influenced positively cushion thickness $(\hat{\mathrm{Y}}=6.223$ $+.014 \mathrm{Pi}+.031 \mathrm{SW})$, leg length $(\hat{\mathrm{Y}}=28.326+.061 \mathrm{Pi}+.05 \mathrm{SW})$ and arm perimeter $(\hat{\mathrm{Y}}=9.173+.053 \mathrm{Pi}+.017 \mathrm{SW})$. Carcass conformation was not alterated by SW.

Key Words: commercial cuts, subcutaneous fat, Longissimus dorsi, empty body weight

\section{Introdução}

Com a estabilização da moeda no país, o produtor de bovinos de corte cada vez mais percebe a necessi- dade de incrementar a lucratividade de sua atividade utilizando-se técnicas mais eficientes, tendo em vista a pequena margem de lucro obtida. Além disso, com o aumento da participação da carne bovina entre os

\footnotetext{
${ }^{1}$ Parte da dissertação de Mestrado do primeiro autor.

2 Zootecnista, Msc. Email: mziegler@terra.com.br

${ }^{3}$ Engenheiro-Agrônomo, PhD, Pesquisador Visitante/CNPq - Departamento de Produção Animal - UFG. E.mail: jorestle@terra.com.br

${ }^{4}$ Eng $^{\circ}$-Agr ${ }^{\circ}$, Msc. Professor Assistente do Departamento de Zootecnia da UFSM.

5 Zootecnista, Msc. Professor Assistente do Departamento de Zootecnia da UFSM.

${ }^{6}$ Zootecnista, Aluno de Doutorado do Programa de Pós-Graduação em Ciência Animal da UFG - Goiânia, GO

${ }^{7}$ Aluno do curso de graduação em Zootecnia da UFSM.
} 
produtos de exportação, o produtor também deve estar atento em produzir carcaças de qualidade.

Nesse sentido, algumas alternativas podem ser utilizadas para incremento da eficiência produtiva, como o uso do confinamento (Restle et al., 2000). Esta técnica é particularmente importante nos sistemas que adotam o ciclo completo, haja vista os benefícios diretos e indiretos. No entanto, para que haja retorno econômico satisfatório, o confinamento deve ser conduzido corretamente, principalmente no sentido de obter a melhor eficiência alimentar.

Dois aspectos são fundamentais na comercialização - o peso dos animais e o grau de acabamento. Para bovinos jovens, no sul do país, as exigências dos frigoríficos são para animais que produzem carcaças acima de $225 \mathrm{~kg}$ e no mínimo $3 \mathrm{~mm}$ de gordura de cobertura. Já na região central do país, carcaças com peso inferior a $240 \mathrm{~kg}$ são penalizadas. A preferência dos frigoríficos por animais de peso mais elevado é que demandam, praticamente, a mesma mão-de-obra e tempo de processamento do que animais mais leves, resultando em menor custo/kg de carcaça beneficiada. Além disso, carcaças mais pesadas produzem músculos maiores, o que é importante na comercialização, para os mercados interno e externo.

$\mathrm{O}$ tecido que sofre maior incremento com o aumento do peso é o adiposo (Berg \& Butterfield, 1976). A gordura que recobre a carcaça é importante, pois reduz as perdas por desidratação durante o resfriamento. A gordura subcutânea também evita o escurecimento externo dos músculos que recobrem a carcaça, conferindo melhor aspecto visual.

Aumentos no rendimento de carcaça em função do avanço do peso de abate são relatados por vários autores (Moody et al., 1970; Zinn et al., 1970; Galvão et al., 1991; Jorge et al., 1999). Segundo Galvão et al. (1991), os rendimentos menores de carcaça em animais mais leves devem-se ao maior peso relativo do couro, das patas e da cabeça. De acordo com Restle et al. (1997a), o aumento no rendimento de carcaça nos animais de peso mais elevado é principalmente conseqüência do grau de acabamento mais adiantado, resultado da maior deposição de gordura na carcaça. Galvão et al. (1991), pesquisando animais Nelore e suas cruzas com raças continentais; Restle et al. (1997 a), novilhos Charolês; e Jorge et al. (1999), diferentes grupos genéticos zebuínos, verificaram incremento na espessura de gordura com o aumento do peso de abate.
Aumento no peso de abate resultou em maior área de músculo Longissimus dorsi, tanto em raças tardias, como o Charolês (Restle et al., 1997a), como em raças precoces, como o Aberdeen Angus (Costa et al., 2002). No entanto, quando expresso por $100 \mathrm{~kg}$ de carcaça, ocorreu decréscimo com o aumento do peso de abate, em ambos grupos genéticos. Por outro lado, Jorge et al. (1999), avaliando as raças Gir, Guzerá, Nelore e Tabapuã, verificaram aumento na área do músculo Longissimus dorsi, porém não constataram decréscimo, quando foi ajustado para $100 \mathrm{~kg}$ de carcaça.

Restle et al. (1997a) enfatizam que o aumento no peso de abate está correlacionado positivamente com importantes características da carcaça rendimento, grau de acabamento, conformação, espessura de coxão e área de Longissimus dorsi. No entanto, aumento no peso de abate pode afetar o desempenho animal (Barber et al., 1981; Restle et al., 1997b; Costa et al., 2002), alterando os custos do processo de terminação e, conseqüentemente, sua lucratividade.

O objetivo deste trabalho foi avaliar as características quantitativas da carcaça de novilhos 5/8 Nelore 3/8 Charolês, terminados em confinamento e abatidos com diferentes pesos.

\section{Material e Métodos}

O estudo foi conduzido no Setor de Bovinocultura de Corte do Departamento de Zootecnia da Universidade Federal de Santa Maria, situada na Depressão Central do Rio Grande do Sul, com coordenadas $29^{\circ} 42^{\prime}$ de latitude Sul e $54^{\circ} 42^{\prime}$ de logitude Oeste, e 96 metros de altitude.

Foram avaliadas as características quantitativas da carcaça de 18 novilhos 5/8 Nelore - 3/8 Charolês castrados, contemporâneos e provenientes do mesmo rebanho, terminados em confinamento e abatidos com 425 (P425), 467 (P467) e 510 kg (P510), sendo os pesos inicialmente pretendidos de 420, 460 e $510 \mathrm{~kg}$.

Durante o confinamento, os animais foram alimentados ad libitum, duas vezes ao dia (8 e 17h), com dieta contendo $10,25 \%$ de proteína bruta e $72,18 \%$ de nutrientes digestíveis totais. A dieta foi calculada segundo o NRC (1996), objetivando ganho de peso médio diário de 1,600 kg/animal, estimando-se consumo de 2,5 kg de matéria seca (MS)/100 kg de peso vivo (PV), sendo a relação volumoso:concentrado de 60:40, com base na MS (Tabela 1).

\section{R. Bras. Zootec., v.33, n.4, p.969-977, 2004}


As pesagens foram realizadas após jejum de sólidos de quatorze horas. Os animais foram abatidos quando atingiram peso de abate próximo ao pretendido. O abate foi realizado em frigorífico comercial, distante 25 km da Fazenda Experimental, seguindo o fluxo normal de abate.

No momento do abate, de cada animal foram tomados os pesos do coração, rins, fígado, baço, pulmões, cabeça, chifres (quando presentes), vassoura da cauda, patas, rúmen + retículo (cheios e vazios), omaso e abomaso (cheios e vazios), intestino delgado + intestino grosso (cheios e vazios), gordura renal, visceral e de toalete, couro e sangue, para determinação do peso de corpo vazio (PCV), que representa o somatório de todos estes componentes mais o peso de carcaça quente subtraído o conteúdo gastrintestinal.

As carcaças foram pesadas no final da linha de abate para determinação do peso de carcaça quente, sendo resfriadas em câmara fria, por 24 horas, à temperatura de 0 a $1^{\circ} \mathrm{C}$. Após esse período de resfriamento, as meia-carcaças foram novamente pesadas para obtenção do peso de carcaça fria e avaliadas subjetivamente quanto à conformação, que representa o grau de musculosidade na região anterior e, principalmente, na região posterior da carcaça, onde estão localizados os cortes mais valorizados; e a maturidade fisiológica, que representa o grau de ossificação das cartilagens torácicas, lombares e entre as vértebras sacrais, segundo metodologia proposta por Müller (1987).

A meia-carcaça fria esquerda foi separada nos cortes comerciais: serrote ou traseiro especial, que compreende a região posterior da carcaça, separado

Tabela 1 - Composição da dieta, base matéria seca Table 1 - Diet composition, dry matter basis

\begin{tabular}{lc}
\hline $\begin{array}{l}\text { Ingredientes } \\
\text { Ingredients }\end{array}$ & $\%$ \\
\hline $\begin{array}{l}\text { Silagem de milho } \\
\text { Corn silage }\end{array}$ & 60,00 \\
$\begin{array}{l}\text { Farelo de trigo } \\
\text { Wheat bran }\end{array}$ & 28,24 \\
$\begin{array}{l}\text { Sorgo grão } \\
\text { Sorghum grain }\end{array}$ & 10,07 \\
$\begin{array}{l}\text { Uréia } \\
\text { Urea }\end{array}$ & 0,17 \\
Calcário calcítico & \\
Limestone & 1,02 \\
NaCl & 0,48 \\
Rumensin & 0,0128 \\
\hline
\end{tabular}

do dianteiro entre a quinta e sexta costelas e do costilhar ou ponta de agulha a uma distância de aproximadamente $20 \mathrm{~cm}$ da coluna vertebral; dianteiro, que compreende o pescoço, paleta, braço e cinco costelas; e costilhar ou ponta de agulha, que compreende a região da sexta costela mais os músculos abdominais. Foram pesados individualmente e determinadas suas proporções em relação à meia-carcaça.

Na meia-carcaça fria direita, foi realizada uma secção na altura da $12^{\mathrm{a}}$ costela, expondo o músculo Longissimus dorsi, sendo traçado em papel vegetal o seu contorno para posterior determinação de sua área $\left(\mathrm{cm}^{2}\right)$ em mesa digitalizadora, utilizando-se o software Siter 1.0. Nesta mesma secção, foi determinada a espessura de gordura subcutânea, pela média aritmética de três observações ao redor do músculo Longissimus dorsi exposto. Ainda na meia-carcaça fria direita, foram feitas as medidas de comprimento de carcaça, tomada do bordo cranial medial da primeira costela e do bordo anterior do osso púbis, comprimento de perna, correspondente à distância entre o bordo anterior do osso púbis e a articulação tíbio-tarsiana; espessura de coxão, medido entre a face lateral e a face medial da porção superior do coxão, com auxílio de um compasso; perímetro e comprimento do braço, de acordo com metodologia descrita por Müller (1987).

O delineamento experimental utilizado foi inteiramente casualizado com três tratamentos e seis repetições. Os dados foram submetidos à análise de regressão e correlação de Pearson, por intermédio do programa estatístico SAS (1997).

O modelo estatístico utilizado foi:

$$
\mathrm{Y}_{\mathrm{ijl}}=\mathrm{B}_{0}+\mathrm{PI}_{\mathrm{l}}+\mathrm{B}_{1} \mathrm{P}_{\mathrm{ij}}+\mathrm{B}_{2} \mathrm{P}^{2} \mathrm{ij}+\mathrm{E}_{\mathrm{ijl}}
$$

em que $\mathrm{Y}_{\mathrm{ijl}}=$ variáveis dependentes; $\mathrm{B}_{0}, \mathrm{~B}_{1}, \mathrm{~B}_{2}=$ parâmetros da equação a serem estimados; $\mathrm{PI}_{1}=$ efeito do peso inicial da repetição “j”; $P_{i j}=$ peso de abate na repetição "j” do tratamento "i”"; $E_{i j l}=$ erro experimental da observação “ijl”, $\operatorname{NID}\left(0, \sigma^{2}\right)$.

\section{Resultados e Discussão}

Os animais permaneceram em confinamento por um período de 30, 65 e 94 dias, respectivamente, nos tratamentos P425, P467 e P510.

Todos os dados apresentados nas tabelas são ajustados para peso inicial. Constam, na Tabela 2, os pesos médios de fazenda e corpo vazio, bem como os

R. Bras. Zootec., v.33, n.4, p.969-977, 2004 
pesos e os rendimentos de carcaça quente e fria em relação aos pesos acima citados e quebra no resfriamento para os diferentes pesos de abate estudados.

Verificou-se que os animais do tratamento P425 produziram carcaças com peso quente de $231,6 \mathrm{~kg}$ e com peso frio de $225,5 \mathrm{~kg}$, acima do mínimo de $225 \mathrm{~kg}$, exigido pelos frigoríficos no sul do país. $\mathrm{O}$ aumento no peso de carcaça foi linear, sendo a diferença do peso de carcaça quente dos animais do tratamento P510, 24,9 e 9,3\% superior aos do P425 e P467, respectivamente.

O rendimento de carcaça quente, calculado em relação ao peso de fazenda, mostrou tendência $(\mathrm{P}=0,1083)$ de aumento frente ao incremento do peso de abate. Já o rendimento de carcaça fria em relação ao peso de fazenda aumentou $(\mathrm{P}=0,0660)$ com o avanço do peso de abate. A maior diferença no rendimento foi entre o P425 e o P467, sendo similar entre o P467 e P510. Aumentos no rendimento de carcaça em função do incremento do peso de abate são relatados por vários autores (Moody et al., 1970; Zinn et al., 1970; Barber et al., 1981; Galvão et al., 1991; May et al., 1992; Jorge et al., 1999). Galvão et al. (1991) relatam que rendimentos menores de carcaça em animais mais leves devem-se ao maior peso relativo do couro, das patas e da cabeça e ao menor teor de gordura na carcaça.

Segundo Restle et al. (1997a), o aumento do rendimento de carcaça nos animais com peso mais elevado é conseqüência, principalmente, do grau de acabamento mais adiantado, em função da maior deposição de gordura na carcaça, o que foi confirmado no presente experimento, pois o rendimento de carcaça fria apresentou correlação positiva com a espessura de gordura de cobertura da carcaça $(r=0,46$; $\mathrm{P}=0,0543)$. Verificou-se, ainda, que o rendimento de

Tabela 2 - Médias ajustadas e erros-padrão para peso final (PVF) e de corpo vazio (PCV), peso de carcaça quente (PCQ) e fria (PCF), rendimento de carcaça quente e fria em relação ao peso final (RCQ e RCF) e em relação ao peso de corpo vazio (RCQCV, RCFCV), e quebra no resfriamento (QR), de acordo com o peso de abate

Table 2 - Adjusted means and standard errors for final weight (FW) and empty body weight (EB), hot (HCW) and cold (CCW) carcass weight, hot and cold carcass dressing percentage in relation to final weight (HDP and CDP) and in relation to EB (HDEB, $C D E B)$, and chilling loss (CL), according to the slaughter weight

\begin{tabular}{|c|c|c|c|c|}
\hline \multirow[t]{2}{*}{$\begin{array}{l}\text { Variáveis } \\
\text { Variables }\end{array}$} & \multicolumn{3}{|c|}{$\begin{array}{l}\text { Peso de abate }(\mathrm{P}), \mathrm{kg} \\
\text { Slaughter weight }(P), \mathrm{kg}\end{array}$} & \multirow[t]{2}{*}{$\begin{array}{l}\text { Equação de regressão } \\
\text { Regression equation }\end{array}$} \\
\hline & 425 & 467 & 510 & \\
\hline $\begin{array}{l}\text { PVF, kg } \\
F W, k g\end{array}$ & $423,9 \pm 7,05$ & $470,5 \pm 7,05$ & $508,7 \pm 7,05$ & $\hat{\mathrm{Y}}=-440,759( \pm 67,598)+1,166( \pm 0,106) \mathrm{Pi}+1,060( \pm 0,121) \mathrm{P}^{\mathrm{a}}$ \\
\hline $\begin{array}{l}\mathrm{PCV}, \mathrm{kg} \\
E B, \mathrm{~kg}\end{array}$ & $367,1 \pm 8,19$ & $408,7 \pm 8,20$ & $459,6 \pm 8,20$ & $\hat{\mathrm{Y}}=-501,690( \pm 78,568)+1,058( \pm 0,123) \mathrm{Pi}+1,155( \pm 0,141) \mathrm{P}^{\mathrm{b}}$ \\
\hline $\begin{array}{l}\mathrm{PCQ}, \mathrm{kg} \\
H C W, \mathrm{~kg}\end{array}$ & $231,6 \pm 5,01$ & $264,8 \pm 5,01$ & $289,3 \pm 5,01$ & $\hat{\mathrm{Y}}=-317,376( \pm 48,708)+0,699( \pm 0,076) \mathrm{Pi}+0,710( \pm 0,087) \mathrm{P}^{\mathrm{c}}$ \\
\hline $\begin{array}{l}\mathrm{RCQ}, \% \\
H D P, \%\end{array}$ & $54,5 \pm 0,69$ & $56,6 \pm 0,69$ & $56,6 \pm 0,69$ & $\hat{\mathrm{Y}}=40,511( \pm 6,925)+0,010( \pm 0,011) \mathrm{Pi}+0,026( \pm 0,012) \mathrm{P}^{\mathrm{d}}$ \\
\hline $\begin{array}{l}\text { RCQCV, \% } \\
H D E B, \%\end{array}$ & $62,8 \pm 0,51$ & $63,6 \pm 0,51$ & $62,7 \pm 0,51$ & $\widetilde{Y}=63,04 \pm 0,009$ \\
\hline $\begin{array}{l}\mathrm{PCF}, \mathrm{kg} \\
C C W, \mathrm{~kg}\end{array}$ & $225,5 \pm 5,00$ & $261,0 \pm 5,01$ & $282,3 \pm 5,01$ & $\hat{\mathrm{Y}}=-312,617( \pm 49,856)+0,672( \pm 0,078) \mathrm{Pi}+0,709( \pm 0,089) \mathrm{P}^{\mathrm{f}}$ \\
\hline $\begin{array}{l}\mathrm{RCF}, \% \\
C D P, \%\end{array}$ & $53,1 \pm 0,66$ & $55,5 \pm 0,66$ & $55,4 \pm 0,66$ & $\hat{\mathrm{Y}}=37,618( \pm 6,793)+0,011( \pm 0,011) \mathrm{Pi}+0,028( \pm 0,012) \mathrm{P}^{\mathrm{g}}$ \\
\hline $\begin{array}{l}\mathrm{RCFCV}, \% \\
C D E B, \%\end{array}$ & $61,4 \pm 0,49$ & $62,3 \pm 0,49$ & $61,3 \pm 0,49$ & $\widetilde{Y}=61,67 \pm 0,009$ \\
\hline $\begin{array}{l}\mathrm{QR}, \% \\
C L, \%\end{array}$ & $2,6 \pm 0,13$ & $2,0 \pm 0,13$ & $2,1 \pm 0,13$ & $\hat{\mathrm{Y}}=5,764( \pm 1,413)-0,003( \pm 0,002) \mathrm{Pi}-0,005( \pm 0,003) \mathrm{P}^{\mathrm{i}}$ \\
\hline
\end{tabular}

${ }^{a} R^{2}=0,9298, P=0,0001 ; b R^{2}=0,9044, P=0,0001 ;{ }^{c} R^{2}=0,9095, P=0,0001 ; d R^{2}=0,2565, P=0,1083 ; R^{2}=0,8402, P=0,0001 ;$ ${ }^{f} R^{2}=0,9018, P=0,0001 ; 9 R^{2}=0,3040, P=0,0660 ; h R^{2}=0,8402, P=0,0001 ;{ }^{i} R^{2}=0,2941, P=0,0734$. 
carcaça fria esteve associado positivamente com a conformação da carcaça $(r=0,49 ; \mathrm{P}=0,0369)$ e com as medidas que avaliam a musculosidade da carcaça, como área do músculo Longissimus dorsi $(\mathrm{r}=0,59$; $\mathrm{P}=0,0101)$ e a espessura de coxão $(\mathrm{r}=0,47 ; \mathrm{P}=0,0469)$.

Quando o rendimento de carcaça foi expresso em relação ao peso de corpo vazio, este não foi influenciado pelo peso de abate, sendo a média para o rendimento de carcaça quente e fria, respectivamente, de 63,04 e 61,67\%. Médias similares sem diferença significativa entre pesos de abate foram relatadas por Jorge et al. (1999), estudando carcaças de zebuínos abatidos com 405, 450 e 500 kg.

A quebra de peso da carcaça durante o resfriamento decresceu $(\mathrm{P}=0,0734)$ com o aumento do peso de abate, em decorrência da menor perda de líquidos durante o resfriamento, porque a gordura que recobre a carcaça atua como isolante, reduzindo as perdas por desidratação (Muller, 1987); fato confirmado, no presente estudo, pela correlação negativa entre espessura de gordura e quebra no resfriamento $(r=-0,38 ; P=0,0119)$. De maneira geral, carcaças com maior espessura de gordura de cobertura apresentam menor quebra durante o resfriamento. Cabe salientar que, em razão de as carcaças do P425 apresentarem, em média, 3,6 mm de espessura de gordura, esta já reduz a perda por desidratação, indicando que maiores correlações seriam esperadas, caso houvesse entre carcaças maior variação quanto à espessura de gordura.

Foi observado que no P425 a quebra durante o resfriamento foi 30 e $24 \%$ superior do que no P467 e P510, respectivamente. O leve incremento na quebra do P510 em relação ao P467 não era esperado, uma vez que os últimos apresentaram espessura de gordura levemente maior. Segundo Restle et al. (1997a), a variação na quebra no resfriamento está associada às oscilações que ocorrem na câmara fria (temperatura, velocidade do vento, número de carcaças). No presente estudo, embora todos os animais tenham sido abatidos no mesmo frigorífico e resfriados na mesma câmara fria, os abates ocorreram em datas diferentes, havendo possibilidade de terem ocorrido oscilações no ambiente da câmara fria. Em seu estudo, Galvão et al. (1991) verificaram que em animais abatidos com peso equivalente a 90, 100 e $110 \%$ do peso adulto, a quebra no resfriamento também decresceu, sendo de 2,55; 1,90 e 1,84\%, respectivamente. Quebras menores que as verificadas no presente experimento são relatadas por Restle et al. (1997a), avaliando carcaças de novilhos Charolês abatidos com 420, 460 e $500 \mathrm{~kg}$.

Na Tabela 3, são apresentados os resultados referentes à espessura de gordura subcutânea e cortes comerciais da carcaça, de acordo com o peso de abate.

A espessura de gordura aumentou linearmente com o avanço do peso de abate; o maior incremento ocorreu do P425 para o P467. As carcaças do P425 apresentaram espessura levemente acima do mínimo exigido pelos frigoríficos ( $3 \mathrm{~mm}$ ) e as do P467 e P510, levemente acima (0,3 e 1,3 mm) do limite superior (6 $\mathrm{mm})$, respectivamente.

Segundo estimativas da equação de regressão, o limite superior de $6 \mathrm{~mm}$ de gordura de cobertura, tolerado pela maioria dos frigoríficos, seria atingido com o peso de abate $465 \mathrm{~kg}$. Já o mínimo de acabamento ( $3 \mathrm{~mm}$ ) seria atingido com peso de abate de $401 \mathrm{~kg}$.

Trabalhando com pesos de abate de 420, 460 e 500 kg, em novilhos Charolês, foram observadas espessuras de gordura inferiores por Restle et al. (1997a) de 2,4; 2,6 e 5,4 mm. Assim como Jorge et al. (1999), trabalhando com novilhos zebuínos com peso de abate de 405,450 e $500 \mathrm{~kg}$, que verificaram espessura de gordura de 2,17; 2,97 e $4 \mathrm{~mm}$, respectivamente.

A espessura de gordura expressa por $100 \mathrm{~kg}$ de carcaça fria e por $100 \mathrm{~kg}$ de peso de corpo vazio aumentaram linearmente com o avanço do peso de abate. Estes resultados refletem a intensificação do processo de deposição de gordura, à medida que os animais se aproximam do tamanho adulto. Aumento na espessura de gordura/100 kg de carcaça fria ocorreu com maior intensidade do P425 ao P467 kg (56\%), caindo para 4\% do P467 ao P510. Redução no incremento da deposição da gordura de cobertura pode ser explicada pelo aumento na deposição da gordura interna (Arboitte et al., 2004b) e pelo aumento da exigência líquida de mantença. Incrementos na espessura de gordura por $100 \mathrm{~kg}$ de corpo vazio, com o aumento no peso de abate, também foram relatados por Galvão et al. (1991) e Jorge et al. (1999), porém bem inferiores aos verificados no presente experimento.

Os pesos dos cortes comerciais serrote, dianteiro e costilhar elevaram-se linearmente, em conseqüência do aumento do peso de abate. Para aumentar em $1 \mathrm{~kg}$ o peso dos cortes serrote, dianteiro e costilhar, realizando-se as estimativas por equação de regressão, foi necessário acréscimo no peso de abate de 6,35; 7,30 e $11,85 \mathrm{~kg}$, respectivamente.

R. Bras. Zootec., v.33, n.4, p.969-977, 2004 
Tabela 3 - Médias ajustadas e erros-padrão para espessura de gordura subcutânea da carcaça (EGS), por 100 kg de carcaça fria (EGS/PCF) e por $100 \mathrm{~kg}$ de peso de corpo vazio (EGS/PCVZ), peso e percentagem do corte serrote, dianteiro e costilhar, de acordo com o peso de abate

Table 3 - Adjusted means and standard errors for subcutaneous fat thickness of the carcass (SFT), per $100 \mathrm{~kg}$ of cold carcass (SFT/CCW) and per $100 \mathrm{~kg}$ of empty body weight (SFT/EB); sawcut, forequarter and sidecut weight and percentage, according to the slaughter weight

\begin{tabular}{|c|c|c|c|c|}
\hline \multirow[t]{2}{*}{$\begin{array}{l}\text { Variáveis } \\
\text { Variables }\end{array}$} & \multicolumn{3}{|c|}{$\begin{array}{l}\text { Peso de abate }(\mathrm{P}), \mathrm{kg} \\
\text { Slaughter weight }(\mathrm{P}), \mathrm{kg}\end{array}$} & \multirow[t]{2}{*}{$\begin{array}{l}\text { Equação de regressão } \\
\text { Regression equation }\end{array}$} \\
\hline & 425 & 467 & 510 & \\
\hline $\begin{array}{l}\text { EGS, mm } \\
\text { SFT,mm }\end{array}$ & $3,6 \pm 0,51$ & $6,3 \pm 0,51$ & $7,3 \pm 0,51$ & $\hat{\mathrm{Y}}=-15,499( \pm 5,159)-0,001( \pm 0,008) \mathrm{Pi}+0,047( \pm 0,009) \mathrm{P}^{\mathrm{a}}$ \\
\hline $\begin{array}{l}\mathrm{EGS} / \mathrm{PCF}, \mathrm{mm} \\
\text { SFT/CCW, } m m\end{array}$ & $1,6 \pm 0,20$ & $2,5 \pm 0,20$ & $2,6 \pm 0,20$ & $\hat{\mathrm{Y}}=-1,724( \pm 2,075)-0,006( \pm 0,003) \mathrm{Pi}+0,013( \pm 0,004) \mathrm{P}^{\mathrm{b}}$ \\
\hline $\begin{array}{l}\mathrm{EGS} / \mathrm{PCV}, \mathrm{mm} \\
S F T / E B, \mathrm{~mm}\end{array}$ & $1,0 \pm 0,12$ & $1,5 \pm 0,12$ & $1,6 \pm 0,12$ & $\hat{\mathrm{Y}}=-1,124( \pm 1,260)-0,003( \pm 0,002) \mathrm{Pi}+0,008( \pm 0,002) \mathrm{P}^{\mathrm{c}}$ \\
\hline $\begin{array}{l}\text { Serrote, kg } \\
\text { Sawcut, } k g\end{array}$ & $57,8 \pm 1,44$ & $66,3 \pm 1,44$ & $70,4 \pm 1,44$ & $\hat{\mathrm{Y}}=-67,132( \pm 14,343)+0,165( \pm 0,022) \mathrm{Pi}+0,157( \pm 0,026) \mathrm{P}^{\mathrm{d}}$ \\
\hline $\begin{array}{l}\text { Serrote, \% } \\
\text { Sawcut, \% }\end{array}$ & $51,5 \pm 0,49$ & $50,1 \pm 0,49$ & $49,4 \pm 0,49$ & $\hat{\mathrm{Y}}=63,007( \pm 4,729)-0,001( \pm 0,007) \mathrm{Pi}-0,026( \pm 0,008) \mathrm{P}^{\mathrm{e}}$ \\
\hline $\begin{array}{l}\text { Dianteiro, kg } \\
\text { Forequarter, kg }\end{array}$ & $42,1 \pm 1,06$ & $48,6 \pm 1,06$ & $53,1 \pm 1,06$ & $\hat{\mathrm{Y}}=-57,442( \pm 10,311)+0,117( \pm 0,016) \mathrm{Pi}+0,137( \pm 0,019) \mathrm{P}^{\mathrm{f}}$ \\
\hline $\begin{array}{l}\text { Dianteiro, \% } \\
\text { Forequarter, \% }\end{array}$ & $37,5 \pm 0,29$ & $36,8 \pm 0,29$ & $37,3 \pm 0,29$ & $\hat{\mathrm{Y}}=37,18 \pm 0,006$ \\
\hline $\begin{array}{l}\text { Costilhar, kg } \\
\text { Sidecut, } \mathrm{kg}\end{array}$ & $12,3 \pm 0,61$ & $17,4 \pm 0,61$ & $19,1 \pm 0,61$ & $\hat{\mathrm{Y}}=-39,132( \pm 6,729)+0,046( \pm 0,011) \mathrm{Pi}+0,084( \pm 0,012) \mathrm{Pg}$ \\
\hline $\begin{array}{l}\text { Costilhar, \% } \\
\text { Sidecut, \% }\end{array}$ & $11,0 \pm 0,38$ & $13,1 \pm 0,38$ & $13,4 \pm 0,38$ & $\hat{\mathrm{Y}}=-3,053( \pm 4,169)+0,005( \pm 0,007) \mathrm{Pi}+0,030(0,007) \mathrm{P}^{\mathrm{h}}$ \\
\hline
\end{tabular}

${ }^{a} R^{2}=0,6331, P=0,0005 ;{ }^{b} R^{2}=0,5107, P=0,0047 ;{ }^{c} R^{2}=0,4942, P=0,0060 ;{ }^{d} R^{2}=0,8597, P=0,0001$; e $R^{2}=0,3948, P=0,0231$;

${ }^{f} R^{2}=0,8783, P=0,0001 ; g R^{2}=0,8196, P=0,0001 ;{ }^{h} R^{2}=0,5241, P=0,0038$.

Comparando o aumento percentual dos cortes em relação ao seu próprio peso, observa-se que aumento do peso de abate do P425 para o P510 resultou em menor acréscimo no serrote (22\%), seguido pelo dianteiro (26\%); o costilhar apresentou o maior incremento (55\%). Costa et al. (2002), avaliando diferentes pesos de abate em animais superprecoce Red Angus, constataram entre os pesos de abate 340 e $430 \mathrm{~kg}$ a mesma hierarquia nos aumentos dos cortes, porém em percentuais menores.

Ainda na Tabela 3, observa-se que, embora tenha ocorrido aumento linear no peso dos cortes comerciais, a proporção relativa na carcaça do corte serrote, onde estão localizados os músculos de maior valor comercial, decresceu, ao passo que a porcentagem do corte costilhar aumentou. Verificou-se, pela estimativa da equação de regressão, que, para cada kg a mais no peso de abate, ocorreu $0,026 \%$ de decréscimo na porcentagem do corte serrote e $0,03 \%$ de acréscimo do corte costilhar; a porcentagem do corte de dianteiro não sofreu alterações. A mudança na proporção dos cortes na carcaça deve-se, em parte, ao ímpeto do crescimento dos músculos.

Segundo Berg \& Butterfield (1976), a maioria dos músculos localizados no corte serrote é de alto a médio ímpeto de crescimento. Além disso, considerando que a porcentagem de dianteiro não foi alterada com o avanço do peso de abate, a queda na porcentagem do serrote foi conseqüência do aumento no peso do costilhar, uma vez que as porcentagens são valores relativos do peso total destes. Vaz (1999) comenta que aumentos na porcentagem de costilhar em carcaças com maior peso e grau de terminação podem ser atribuídos à maior deposição de gordura nesta área. No presente trabalho, a correlação entre a espessura de gordura e o peso do costilhar foi $0,71(\mathrm{P}=0,0009)$ e com a porcentagem de costilhar, de $0,82(\mathrm{P}=0,0001)$. Correlação positiva entre as mesmas variáveis foi observada por Costa et al. (2002), porém em magnitudes menores.

\section{R. Bras. Zootec., v.33, n.4, p.969-977, 2004}


Na Tabela 4, constam as médias referentes às características métricas da carcaça e aquelas relacionadas com sua musculosidade.

Ao avaliar as medidas referentes ao comprimento de carcaça e de perna, constata-se que os animais ainda estavam em crescimento corporal. A equação de regressão para crescimento indica que, para cada $\mathrm{kg}$ a mais no peso de abate, houve aumento de $0,075 \mathrm{~cm}$ no comprimento de carcaça e $0,05 \mathrm{~cm}$ no comprimento de perna. Quando os dados foram expressos em relação a $100 \mathrm{~kg}$ de carcaça fria e $100 \mathrm{~kg}$ de corpo vazio, fica evidente que o crescimento corporal desacelerava, à medida que os animais eram abatidos com maior peso.

Tabela 4 - Médias ajustadas e erros-padrão para comprimento de carcaça (CCarc) e perna (CPerna), CCarc e CPerna expressos por $100 \mathrm{~kg}$ de carcaça fria (CCarc/PCF e CPerna/PCF) e de corpo vazio (CCarc/PCV e CPerna/PCV), área do músculo Longissimus dorsi (ALD), ALD por $100 \mathrm{~kg}$ de carcaça fria (ALD/PCF), ALD por $100 \mathrm{~kg}$ de peso de corpo vazio (ALD/PCV), perímetro de braço (PerBraço), espessura de coxão (EspCox), conformação (Conf) da carcaça e maturidade fisiológica (MatFis), de acordo com o peso de abate

Table 4 - Adjusted means and standard errors for carcass (CL) and leg length, CL and leg length per $100 \mathrm{~kg}$ of cold carcass (CL/CCW and leg length/CCW), CL and leg length per $100 \mathrm{~kg}$ of empty body weight (CL/EB and leg length/EB), Longissimus dorsi muscle area (LDA), LDA per $100 \mathrm{~kg}$ of cold carcass(LDA/CCW), ALD per $100 \mathrm{~kg}$ of empty body weight (LDA/EB), arm perimeter, cushion thickness, carcass conformation (Conf) and physiological maturity (PhyMat), according to the slaughter weight

\begin{tabular}{|c|c|c|c|c|}
\hline \multirow[t]{2}{*}{$\begin{array}{l}\text { Variáveis } \\
\text { Variables }\end{array}$} & \multicolumn{3}{|c|}{$\begin{array}{l}\text { Peso de abate }(\mathrm{P}), \mathrm{kg} \\
\text { Slaughter weight }(P), \mathrm{kg}\end{array}$} & \multirow[t]{2}{*}{$\begin{array}{l}\text { Equação de regressão } \\
\text { Regression equation }\end{array}$} \\
\hline & 425 & 467 & 510 & \\
\hline $\begin{array}{l}\text { CCarc, } \mathrm{cm} \\
C L, \mathrm{~cm}\end{array}$ & $122,9 \pm 1,31$ & $126,1 \pm 1,31$ & $128,9 \pm 1,31$ & $\hat{\mathrm{Y}}=58,564( \pm 12,424)+0,092( \pm 0,020) \mathrm{Pi}+0,075( \pm 0,022) \mathrm{P}^{\mathrm{a}}$ \\
\hline $\begin{array}{l}\mathrm{CCarc} / \mathrm{PCF}, \mathrm{cm} \\
C L / C C W, \mathrm{~cm}\end{array}$ & $56,0 \pm 1,17$ & $48,4 \pm 1,17$ & $46,1 \pm 1,17$ & $\hat{\mathrm{Y}}=154,338( \pm 12,407)-0,131( \pm 0,019) \mathrm{Pi}-0,124( \pm 0,022) \mathrm{P}^{\mathrm{b}}$ \\
\hline $\begin{array}{l}\mathrm{CCarc} / \mathrm{PCV}, \mathrm{cm} \\
C L / E B, \mathrm{~cm}\end{array}$ & $34,0 \pm 0,51$ & $30,2 \pm 0,51$ & $28,2 \pm 0,51$ & $\hat{\mathrm{Y}}=86,560( \pm 5,209)-0,063( \pm 0,008) \mathrm{Pi}-0,0716( \pm 0,009) \mathrm{P}^{\mathrm{C}}$ \\
\hline $\begin{array}{l}\text { CPerna, } \mathrm{cm} \\
\text { Leg length, } \mathrm{cm}\end{array}$ & $70,9 \pm 0,73$ & $73,8 \pm 0,73$ & $74,9 \pm 0,73$ & $\hat{\mathrm{Y}}=28,326( \pm 7,173)+0,061( \pm 0,011) \mathrm{Pi}+0,050( \pm 0,013) \mathrm{P}^{\mathrm{d}}$ \\
\hline $\begin{array}{l}\text { CPerna/PCF, cm } \\
\text { Leg length/CCW, cm }\end{array}$ & $32,0 \pm 0,71$ & $28,3 \pm 0,71$ & $26,8 \pm 0,71$ & $\hat{\mathrm{Y}}=81,810( \pm 7,105)-0,065( \pm 0,011) \mathrm{Pi}-0,064( \pm 0,013) \mathrm{P}^{\mathrm{e}}$ \\
\hline $\begin{array}{l}\mathrm{CPerna} / \mathrm{PCV}, \mathrm{cm} \\
\text { Leg length/EB, cm }\end{array}$ & $19,6 \pm 0,45$ & $18,0 \pm 0,45$ & $16,4 \pm 0,45$ & $\hat{\mathrm{Y}}=47,366( \pm 4,254)-0,031( \pm 0,007) \mathrm{Pi}-0,039( \pm 0,008) \mathrm{P}^{\mathrm{f}}$ \\
\hline $\begin{array}{l}\text { ALD, } \mathrm{cm}^{2} \\
L D A, \mathrm{~cm}^{2}\end{array}$ & $59,0 \pm 2,81$ & $66,6 \pm 2,81$ & $68,6 \pm 2,81$ & $\hat{\mathrm{Y}}=-33,471( \pm 27,436)+0,120( \pm 0,043) \mathrm{Pi}+0,120( \pm 0,049) \mathrm{Pg}$ \\
\hline $\begin{array}{l}\text { ALD/PCF, } \mathrm{cm}^{2} \\
L D A / C C W, \mathrm{~cm}^{2}\end{array}$ & $26,3 \pm 0,84$ & $25,5 \pm 0,84$ & $24,3 \pm 0,84$ & $\hat{\mathrm{Y}}=45,173( \pm 8,011)-0,023( \pm 0,013) \mathrm{Pi}-0,028( \pm 0,014) \mathrm{P}^{\mathrm{h}}$ \\
\hline $\begin{array}{l}\mathrm{ALD} / \mathrm{PCV}, \mathrm{cm}^{2} \\
L D A / E B, \mathrm{~cm}^{2}\end{array}$ & $16,2 \pm 0,61$ & $15,9 \pm 0,61$ & $15,4 \pm 0,61$ & $\hat{\mathrm{Y}}=14,8 \pm 0,010$ \\
\hline $\begin{array}{l}\text { PerBraço, cm } \\
\text { Arm perimeter, } \mathrm{cm}\end{array}$ & $37,0 \pm 1,12$ & $34,2 \pm 1,12$ & $38,4 \pm 1,12$ & $\hat{\mathrm{Y}}=9,173( \pm 12,961)+0,053( \pm 0,020) \mathrm{Pi}+0,017( \pm 0,023) \mathrm{P}^{\mathrm{i}}$ \\
\hline $\begin{array}{l}\text { EspCox, cm } \\
\text { Cushion thickness,cm }\end{array}$ & $24,8 \pm 0,78$ & $25,2 \pm 0,78$ & $27,3 \pm 0,78$ & $\hat{\mathrm{Y}}=6,223( \pm 7,672)+0,014( \pm 0,012) \mathrm{Pi}+0,031( \pm 0,014) \mathrm{P}^{\mathrm{j}}$ \\
\hline $\begin{array}{l}\text { Conf }{ }^{1} \text {, pontos } \\
\text { Conf, points }\end{array}$ & $10,5 \pm 0,39$ & $11,0 \pm 0,39$ & $10,7 \pm 0,39$ & $\hat{\mathrm{Y}}=10,7 \pm 0,007$ \\
\hline $\begin{array}{l}\text { MatFis, pontos } \\
\text { PhyMat, points }\end{array}$ & $13,3 \pm 0,19$ & $13,5 \pm 0,19$ & $13,3 \pm 0,19$ & $\hat{\mathrm{Y}}=13,4 \pm 0,003$ \\
\hline
\end{tabular}

a $R^{2}=0,6886, P=0,0002 ;{ }^{b} R^{2}=0,8362, P=0,0001 ;{ }^{c} R^{2}=0,8884, P=0,0001 ;{ }^{d} R^{2}=0,7483, P=0,0001 ;$ e $R^{2}=0,7981 ; P=0,0001$;

${ }^{f} R^{2}=0,7646, P=0,0001 ;{ }^{g} R^{2}=0,4781, P=0,0076 ;{ }^{h} R^{2}=0,2996, P=0,0692 ;{ }^{i} R^{2}=0,3348, P=0,0470 ;{ }^{j} R^{2}=0,3055, P=0,0650$.

1 Conformação: $10=$ boa -; 11= boa; $12=$ boa + (Conformation: $10=$ good -; $11=$ good; $12=$ good + ).

\section{R. Bras. Zootec., v.33, n.4, p.969-977, 2004}


Verificou-se também que a queda no comprimento de perna, quando expresso por $100 \mathrm{~kg}$ de carcaça fria, foi mais acentuada que a queda do comprimento de carcaça.

A medida mais utilizada na avaliação do desenvolvimento muscular da carcaça é a área do músculo Longissimus dorsi (ALD). Segundo estimativa da equação de regressão, a ALD aumentou $0,12 \mathrm{~cm}^{2}$ a cada kg de aumento no peso de abate. O maior incremento na ALD (13\%) ocorreu quando o peso de abate aumentou do P425 para o P467, bem superior aos 3\%, quando o peso de abate aumentou do P467 ao P510.

Ao ajustar ALD para $100 \mathrm{~kg}$ de carcaça fria, ocorreu decréscimo de $0,03 \mathrm{~cm}^{2}$ para cada $\mathrm{kg}$ no aumento no peso de abate, comportamento também constatado por Restle et al. (1997a). Segundo esses autores, o decréscimo decorre da redução do nível de desenvolvimento muscular, à medida que aumenta o peso dos novilhos. Costa et al. (2002), trabalhando com animais Aberdeen Angus abatidos com pesos crescentes, relataram decréscimo superior ao deste experimento, no valor de $0,04 \mathrm{~cm}^{2} / 100 \mathrm{~kg}$ de carcaça fria. Maldonado et al. (2002) encontram valores para ALD/100 kg de 28,39; 22,22 e 21,65 $\mathrm{cm}^{2}$, respectivamente, para animais abatidos com 430, 470 e $530 \mathrm{~kg}$, observando-se diferença significativa entre os novilhos abatidos com 430 em relação aos 470 e 530, que não diferiram entre si.

A ALD ajustada/100 kg de PCV não foi influenciada pelo peso de abate, apresentando valor médio para os três tratamentos de $14,84 \mathrm{~cm}^{2}$, bem próximo ao relatado por Jorge et al. (1999), de 13,86 $\mathrm{cm}^{2}$.

O comportamento do decréscimo do desenvolvimento do músculo Longissimus dorsi, quando expresso por $100 \mathrm{~kg}$ de carcaça, foi inverso ao verificado com a espessura de gordura, indicando a desaceleração da formação do tecido muscular e a intensificação da deposição do tecido adiposo, o que é esperado quando o animal se aproxima do peso adulto (Berg \& Butterfield, 1976).

Outras medidas relacionadas com 0 desenvolvimento muscular da carcaça são a espessura de coxão e o perímetro de braço, que aumentaram linearmente com o avanço do peso de abate. As estimativas obtidas por meio da equação mostram que os aumentos foram de 0,03 e $0,02 \mathrm{~cm}$, respectivamente, para cada $\mathrm{kg}$ a mais no peso de abate. Ambas as medidas foram correlacionadas positiva- mente com o ALD, sendo a correlação com a espessura de coxão de $0,56(\mathrm{P}=0,0158)$ e com perímetro de braço de $0,55(\mathrm{P}=0,0176)$.

A conformação que mede subjetivamente a expressão de cobertura muscular da carcaça não foi influenciada pelo peso de abate, sendo as carcaças classificadas como "boa típica”. Segundo Müller (1987), carcaças com melhor conformação tendem a apresentar menor proporção de osso e maior porção comestível, o que foi constatado no presente trabalho, sendo a relação entre conformação e porcentagem de osso negativa ( $r=-0,27)$, embora não-significativa $(P=0,2790)$. As correlações entre conformação, perímetro de braços e espessura de coxão foram positivas, embora não significativas $(r=0,33$; $\mathrm{P}=0,1859 ; \mathrm{r}=0,37 ; \mathrm{P}=0,1202$; respectivamente). Já Costa et al. (2002) verificaram correlações maiores e significativas entre conformação e medidas que avaliam o desenvolvimento muscular da carcaça.

A maturidade fisiológica das carcaças, avaliada subjetivamente pela ossificação das cartilagens presentes nos processos espinhosos das vértebras torácicas, lombares e entre as vértebras sacrais, não foi influenciada pelo peso de abate. A diferença de tempo de abate entre animais mais leves e mais pesados (64 dias) não foi suficiente para alterar significativamente o grau de ossificação dos processos espinhosos das vértebras. Costa et al. (2002) também constataram que a maturidade fisiológica das carcaças não foi alterada, quando os novilhos foram terminados em confinamento com diferentes pesos de abate.

\section{Conclusões}

O rendimento de carcaça fria aumentou com o avanço do peso de abate, mas não foi alterado quando expresso em relação ao corpo vazio.

Aumento no peso de abate acarretou alterações nas medidas que avaliam o desenvolvimento do tecido muscular e adiposo. Embora a área do músculo Longissimus dorsi tenha aumentado com o peso de abate, houve redução quando ajustado para $100 \mathrm{~kg}$ de carcaça. Já a espessura de gordura da carcaça aumentou com o avanço do peso de abate, mesmo quando ajustada para $100 \mathrm{~kg}$ de carcaça.

Aumento do peso de abate resultou em redução da porcentagem do corte serrote e acréscimo linear da porcentagem do corte costilhar na carcaça. 


\section{Literatura Citada}

ARBOITTE, M.Z.; RESTLE, J.; ALVES FILHO, D.C. et al. Desempenho em confinamento de novilhos 5/8 Nelore - 3/8 Charolês abatidos em diferentes estádios de desenvolvimento. Revista Brasileira de Zootecnia, v.33, n.4, 2004a.

ARBOITTE, M.Z.; RESTLE, J.; ALVES FILHO, D.C. et al. Composição física da carcaça, qualidade da carne e conteúdo de colesterol no músculo Longissimus dorsi de novilhos 5/8 Nelore - 3/8 Charolês abatidos em três estádios de desenvolvimento. Revista Brasileira de Zootecnia, v.33, n.4, 2004b.

BARBER, K.A.; WILSON, L.L.; ZIEGLER, J.H. et al. Charolais and Angus steers slaughtered at equal percentages of mature cow weight. I. Effects of slaughter weight and diet energy density on carcass traits. Journal of Animal Science, v.52, n.2, p.218-231, 1981

BERG, R.T.; BUTTERFIELD, R.M. New concepts of cattle growth. 1.ed. New York, 1976. 240p.

COSTA, E.C.; RESTLE, J.; VAZ, F.N. et al. Características da carcaça de novilhos Red Angus superprecoces abatidos com diferentes pesos. Revista Brasileira de Zootecnia, v.31, n.1, p.119-128, 2002

DI MARCO, O.N. Crescimento y respuesta animal. Buenos Aires: Associaccíon Argentina de Producción Animal, 1994. 129p.

GALVÃO, J.G.; FONTES, C.A.A.; PIRES, C.C. et al. Características e composição da carcaça de bovinos nãocastrados, abatidos em três estágios de maturidade (estudo II) de três grupos raciais. Revista da Sociedade Brasileira de Zootecnia, v.20, p.502-512, 1991.

JORGE, A.M.; FONTES, C.A.A.; PAUlinO, M.F. et al. Desempenho produtivo de animais de quatro Raças Zebuínas, abatidos em três estádios de maturidade. 2. Características da carcaça. Revista Brasileira de Zootecnia, v.28, p.381-387, 1999.

MALDONADO, F.; ALLEONI, G.F.; QUEIROZ, A.C. et al. Características da carcaça de bovinos de três grupos genéticos terminados em confinamento e abatidos em três categorias de peso. In: REUNIÃO ANUAL DA SOCIEDADE BRASILEIRA DE ZOOTECNIA, 39., 2002, Recife. Anais... Recife: Sociedade Brasileira de Zootecnia/ Technomedia,[2002]. CD-ROM. Nutrição de Ruminantes. $06 S B Z 1326$.
MAY, S.G.; DOLEZAL, H.G.; GILL, D.R. et al. Effects of days fed, carcass grade traits, and subcutaneous fat removal on postmortem muscle characteristics and beef palatability. Journal of Animal Science, v.70, p.444-453, 1992.

MORENO, J.A. Clima do Rio Grande do Sul. Porto Alegre: Secretaria da Agricultura, 1961. 41p.

MOODY, W.G.; LITTLE, J.E. Jr.; THRIFT, F. A. et al. Influence of length of feeding a high roughage ratio on quantitative and qualitative characteristics of beef. Journal of Animal Science, v.31, p.866-873, 1970.

MÜLLER, L. Normas para avaliação de carcaças e concurso de carcaça de novilhos. 2.ed. Santa Maria: Universidade Federal de Santa Maria, 1987. 31p.

NATIONAL RESEARCH COUNCIL - NRC. Nutrient requirements of domestic animals. 7.rev.ed. Washington, D.C.: National Academy Press, 1996. 242p.

RESTLE, J.; KEPLIN, L.A.S.; VAZ, F.N. Características quantitativas da carcaça de novilhos Charolês, abatidos com diferentes pesos. Pesquisa Agropecuária Brasileira, v.32, n.8, p.851-856, 1997a

RESTLE, J.; KEPLIN, L.A.S.; VAZ, F.N. Desempenho em confinamento de novilhos Charolês terminados com diferentes pesos. Pesquisa Agropecuária Brasileira, v.32, n.8, p.857-860, 1997b.

RESTLE, J.; VAZ, F.N.; FEIJÓ, G.L.D. et al. Características da carcaça de bovinos de corte inteiros ou castrados de diferentes composições raciais Charolês e Nelore. Revista Brasileira de Zootecnia, v.29, n.5, p.1371-1379, 2000.

STATISTICAL ANALYSES SYSTEM - SAS. SAS/STAT. User's guide statistics. 4.ed. Cary: 1997. v.2. 943p.

VAZ, F.N. Cruzamento alternado das raças Charolês e Nelore: características de carcaça e da carne de novilhos abatidos aos dois anos. Santa Maria: Universidade Federal de Santa Maria, 1999. 58p. Dissertação (Mestrado em Zootecnia) - Universidade Federal de Santa Maria, 1999.

ZINN, D.W.; DURRHAN, R.M.; HEDRICK, H.B. Feedlot and carcass grade characteristics of steers and heifers as influenced by days on feed. Journal of Animal Science, v.31, p.302-306, 1970. 